\title{
Euthanasia for Children with Cancer: A Policy Brief
}

\author{
Abdulaziz Thaer Aziz Khader ${ }^{1}$, Majd T. Mrayyan², \\ ${ }^{I} M S N c$, RN, Oncology Nursing Master's Student, Faculty of Nursing, The Hashemite University, Jordan \\ abd_abuthaer@yahoo.com \\ ${ }^{2}$ PhD, RN, Professor, Consultant of Nursing, Dean of Scientific Research, The Hashemite University, Jordan \\ mmrayyan@hu.edu.jo
}

\begin{abstract}
Euthanasia is one of the problems that have been much debated, especially for the hopeless patients, such as children with cancer in advance stage. The current policy applied in the Medical Jordanian Constitution does not differentiate between active and passive euthanasia and it is general for all patients without regard to the restriction of age group, the special situation of children with cancer and developing alternative methods such as palliative care. The recommendations proposed in this paper are prohibiting active euthanasia, enhancing and developing special palliative care units for children with cancer and allowing passive euthanasia and palliative care to work together within the limits of the law.
\end{abstract}

Keywords: euthanasia, passive, active, palliative care, cancer, children

\section{Executive Summary}

A policy brief is a document which outlines the rationale for choosing a particular policy alternative or course of action in a current policy debate ("Policy", n.d). Euthanasia means "good death" and intended for active, intentional ending the life of patients with hopeless conditions by a physician (Danielle, Perez-Cruz, \& Craig, 2011). There are two types of euthanasia; passive when allowing a patient to die by withholding treatment, whereas active euthanasia when taking measures to directly cause a patient's death.

Euthanasia is one of the problems that has been much debated between the proponents and opponents in medical and legal fields, where is the debate about several aspects such as: moral and religion stance, the right of the patient, the responsibility of medicine, personal relationship and society, the effectiveness of alternative methods such as palliative care and with respect to children; many articles discussed the competency of decision and the need for adult to protect them (Kelly, 2014). The current policy applied in the Medical Jordanian Constitution dose not differentiate between active and passive euthanasia or consider the variety of age groups and the unique situation of children with cancer.

The purpose of this brief is to discuss the current policy statement regarding euthanasia for children and recommend alternative approaches for ending life in a peaceful and dignified matter.

\section{Context and Importance of the Problem}

Euthanasia is considered one of the controversial issues in the legal and medical constitutions, some western countries allow it for patients -with hopeless conditions- who are interested as long as they have exceeded 18 years olds (Pereira, 2011), on the other hand, some countries allow euthanasia for minors if they are terminally ill, have great pain, and no treatment to alleviate their distress, with the consent of a competent child, his parents and patient's medical team (Carter, 2014).

In Islamic countries -because of the predominant religious view compared to secular Western countries- active euthanasia is rejected with some discussion and debate about the acceptance of passive euthanasia for some medical conditions (Aghababaei, Hatami, \& Rostami, 2011). For example, in Jordan -as an Islamic country- active and passive euthanasia are considered medical practices punishable by law as an intentional act even if informed consent is taken from the patient or his family (Naga \& Mrayyan, 2013).

Nowadays, while most of incurable diseases are being treated, many diseases, such as cancer, which impose great pain and suffering upon the patients are still common (Mousavi, Akbari, Kashani, Akbari, \& Sepas, 2011). Cancer patients are considered to be one of the most deaths by euthanasia (Smets, Bilsen, Cohen, Rurup \& Deliens, 2010). About 1 out of 10 end-of-life cancer deaths is hastened through euthanasia or physician assisted suicide (Ruijs, Kerkhof, Wal, \& Onwuteaka-Philipsen, 2011). In the Netherlands -where the infant euthanasia is legal- estimated 10 to 15 cases of infant euthanasia actually happened, but only about a fifth were reported (Nuwer, 2014), the research evidence remains scarce regarding the euthanasia for minors with cancer (Kelly, 2014).

Euthanasia is one of the methods alleviate the suffering of patients, especially terminally ill children with cancer, but the Jordanian law does not provide immunity and protection to those who carry out euthanasia, 
in fact, there are many issues encountered their application in Jordan and made prohibition policy for all types of euthanasia.

Preventing and prohibiting euthanasia policy in Jordan is because of several reasons; the majority of the Muslim community rejects euthanasia because the Holy Quran as the fundamental reference of Islamic law prohibits euthanasia (Mousavi et al., 2011). Some believe that euthanasia is morally unacceptable, especially when it extends to minors (Bovens, 2015), and others believe that children are not capable of discerning and look at them as a vulnerable group and may be forced into seeking death, also extending the law to children will be seen by some as being patent evidence of that 'slippery slope' in action (Samanta, 2015).

In fact, all the debates about preventing euthanasia in Jordan is intended for the active type not a passive, accordingly, it can allow for this type of euthanasia to apply in Jordan hospitals for the children with advanced cancer and hopeless condition and make it legal.

The patient's right to choose the appropriate model of treatment, including refraining from taking the treatment, the general prohibition to the all types of euthanasia in Jordan, including passive euthanasia, is considered deprives the patient of his right to self-determination and do not allow to the medical team and patient family to choose what is appropriate for their child's condition. On the other, hand the palliative care, as an alternative method of euthanasia, in Jordan, is started at King Hussein Cancer Center (KHCC) and does not appear in other health care institutions, this prevent children with cancer who do not go to choose passive euthanasia from their right to access to palliative care service.

\section{Critique of Policy Options}

The active euthanasia applied in some western countries for adult patients, but there are many arguments about the extension of the law for pediatric patients, on the opposite side in Islamic countries active euthanasia is prohibited, for example, in our case the Jordanian law prohibits both active and passive euthanasia because of the conflict between the concept of euthanasia and Islamic values.

The policy that punishes the practice of active euthanasia is compatible with the current situation in Jordan when we consider the prohibition of such practices to be constant with Islamic values. In addition, the role of medicine is to heal and cure not to hasten death, for that there is a need to develop and create the culture of palliative care that are directed to children with cancer and family alike.

The role of palliative care for children with cancer is very important and should be available for each patient when we prohibit the active euthanasia, because the healing of cancer patients may take place even when a cure is not obtained and the permanent suffering of these patients make such type of cure is very crucial. Moreover, the public funding for palliative care must be increased and guarantee the right to palliative care in every hospital, nursing home, and even at home.

In comparison, between the euthanasia and palliative treatment; an ethos that focuses on palliative care, but that allows the possibility that death might be hastened is preferable to one that focuses on hastening death.

Actually, the prevention of all types of euthanasia policy needs to be adjusted by allowing for the passive type of euthanasia to be applied when the multidisciplinary medical team decides that the patient condition is hopeless with the presence of request taken from the competent children and his family.

The Passive euthanasia is considered one of the methods that could be used to alleviate the suffering of patients and it does not conflict with the religion perspective, according to the Islamic Fiqh Academy in Maka Almokrma (2015); passive euthanasia is allowed and legalized in Islam when the doctor decides that a patient's condition is hopeless. Depriving the patients from their right to self-determination and freedom of choice to suit options for their health status. The development of a law that allows passive euthanasia, as a choice of treatment options, has to be implemented; giving immunity and protection to the child's family and the care providers when they decide to choose passive euthanasia, in addition to reduce the cost of treatment in what is useless.

The American Academy of Pediatrics (2007) "recommends making treatment decisions that are guided primarily by the best interest of the child", this means the deprive of the competent child from his right to choose what he want as a method of his treatment, such as passive euthanasia, it is a clear violation of a child's right. In order to assess the child competency and capacity for discernment that must he undergo to assessment by a psychiatrist or psychologist (Hanson, 2015). Furthermore, the decisions of multidisciplinary medical team and child's family about passive euthanasia, it is exactly the same way that they make all other medical decisions for a child patient.

When authorizing passive euthanasia, the awareness level of the child's family and care givers needs to be increased to protect them from making a bad choice. Medical ethics and the role of medicine in the patient's care do not contradict with the passive euthanasia for children with cancer. The existence of a contradiction between ethics, medical responsibility and euthanasia is directed towards the active type not the passive, in conclusion, passive euthanasia and palliative care can work together. 


\section{Recommendations}

- Enforce a law prohibiting the practice of active euthanasia for the hopeless patients and children with end stage cancer, because of the conflict and inconsistency between active euthanasia and both of Islamic values and Jordanian culture, and the strict legal measures should be taken to punish those who carry out active euthanasia.

- Prioritize palliative care as an alternative method for active euthanasia and encourage the development and bulid-up of specialized units to provide palliative care for children with cancer in all hospitals that provide a medical service for cancer patients and their families and make these units available to all children who need it.

- Conduct an orientation and education program for children with cancer, families and care providers about the palliative care and provide them with all the information with easy access and consultation of all medical and social disciplines about this issue.

- Provide a financial support to establish the care according to international standards of palliative care for children with end stage cancer.

- Formulate a new policy to permit the practice of passive euthanasia and make it an available choice for patients, especially for children with end stage cancer to alleviate the suffering when the multidisciplinary medical team decides that the patient's condition is hopeless. Informed consent should be taken from the child and his family after being assessed by a psychiatrist or psychologist; to determine he is competent and capable of being discerned with no force on him. This method is not conflicted with Islamic religion and it is consistent with the cultural values in Jordan.

- Motivate the academic organization such as universities and continuing education institutions in hospitals to pay more attention to the studying of medical ethics for all affiliate members, because there is a strong relationship between the concept of euthanasia and medical ethics.

Finally, the palliative care and passive euthanasia can work together and could replace active euthanasia, the researchers wish from the policy makers to take into consideration the previous recommendations listed in this brief.

\section{Sources Recommended}

Hanson, S. S. (2015). Pediatric Euthanasia and Palliative Care Can Work Together. American Journal of Hospice and Palliative Medicine, 33(5), 421-424. doi:10.1177/1049909115570999

\section{References}

[1]. Aghababaei N., Hatami J., Rostami R. (2011). The role of individual characteristics and judgment pattern in attitude toward euthanasia. Iranian Journal of Critical Care Nursing, 4 (1) :23-32.

[2]. American Academy of Pediatrics. Noninitiation or withdrawal of intensive care for high-risk newborns. Pediatrics. 2007;119(2): 401-403.

[3]. Bovens, L. (2015). Child euthanasia: Should we just not talk about it? J Med Ethics Journal of Medical Ethics, 41(8), 630-634. doi:10.1136/medethics-2014-102329

[4]. Carter, B. S. (2014). Why Palliative Care for Children is Preferable to Euthanasia. American Journal of Hospice and Palliative Medicine, 33(1), 5-7. doi:10.1177/1049909114542648

[5]. Danielle, K. N., Pedro, P. P., \& Craig, B. D. (2011). Ethical Issues in Palliative Care. Primary Care, 1-11. doi:10.1016/j.pop.2011.03.003

[6]. Hanson, S. S. (2015). Pediatric Euthanasia and Palliative Care Can Work Together. American Journal of Hospice and Palliative Medicine, 33(5), 421-424. doi:10.1177/1049909115570999

[7]. Kelly, D. (2014). Euthanasia for children and young people? International Journal of Palliative Nursing, $20(5), 211-211$. doi:10.12968/ijpn.2014.20.5.211

[8]. Khadmi, ND. (2015). Euthanasia and stop the treatment for the hopeless patient the Legitimacy and judgment. Islamic Fiqh Academy in Maka Almokrma, 22: 1-36.

[9]. Mousavi SM., Akbari A., Kashani F., Akbari ME., Sepas H. (2011) Euthanasia in Cancer Patients, Islamic Point of View. Iranian Journal of Cancer Prevention.

[10]. Naga, B. S., \& Maryyan, M. T. (2013). Legal and Ethical Issues of Euthanasia : Argumentative Essay. Middle East Journal of Nursing MEJN, 7(5), 31-39. doi:10.5742/mejn.2013.75330

[11]. News \& Announcements. (n.d.). Retrieved November 15, 2016, from http://www.policy.hu/

[12]. Nuwer R. (2014) Mercy for all?. NewScientist.

[13]. Pereira, J. (2011). Legalizing euthanasia or assisted suicide: The illusion of safeguards and controls. Current Oncology, 18(2). doi:10.3747/co.v18i2.883

[14]. Ruijs, C. D., Kerkhof, A. J., Wal, G. V., \& Onwuteaka-Philipsen, B. D. (2011). Depression and explicit requests for euthanasia in end-of-life cancer patients in primary care in the Netherlands: A longitudinal, prospective study. Family Practice, 28(4), 393-399. doi:10.1093/fampra/cmr006

[15]. Samanta J.(2015) Children and euthanasia: Belgium's controversial new law. Diversity and Equality in Health and Care, 12(1): 4-5

[16]. Smets, T., Bilsen, J., Cohen, J., Rurup, M. L. \& Deliens, L. (2010). Legal euthanasia in Belgium: characteristics of all reported euthanasia cases. Medical Care Journal, 48 (2), 187-192. Doi: 10.1097/MLR.0b013e3181bd4dde 